TIFR/TH/91-37

August, 1991

\title{
TWISTED BLACK $p$-BRANE SOLUTIONS IN STRING THEORY
}

\author{
ASHOKe SEN * \\ Tata Institute of Fundamental Research, Homi \\ Bhabha Road, Bombay 400005, India
}

\begin{abstract}
It has been shown that given a classical background in string theory which is independent of $d$ of the space-time coordinates, we can generate other classical backgrounds by $O(d) \otimes O(d)$ transformation on the solution. We study the effect of this transformation on the known black p-brane solutions in string theory, and show how these transformations produce new classical solutions labelled by extra continuous parameters and containing background antisymmetric tensor field.

Prefitem
\end{abstract}

^ e-mail address: SEN@TIFRVAX.BITNET 
In a previous paper [1] we showed that in string theory, if we have an exact classical solution which is independent of $d$ of the space-time coordinates, then we can perform an $O(d) \otimes O(d)$ transformation on the solution, which produces a new configuration of string field, satisfying the classical equations of motion to all orders in the string tension $\alpha^{\prime}$. This generalised the result found by Meissner and Veneziano [2] [3] to leading order in $\alpha^{\prime}$. In this paper we shall study the effect of this $O(d) \otimes O(d)$ transformation on the known black $p$-brane solutions in string theory, and obtain new classical solutions in string theory labelled by extra continuous parameters.

We begin by recalling the general argument of ref.[1] and also by giving a generalised version of the analysis of ref.[3]. In the language of string field theory, looking for solutions which are independent of $d$ of the coordinates (say $Y^{i}$, $1 \leq i \leq d)$ corresponds to looking for a string state $|\Psi\rangle$ carrying zero momentum in these $d$ directions. Restricting string states of this type gives us the reduced string field theory action which governs the classical dynamics in this subspace. This reduced action, in turn, is expressed in terms of correlation functions of vertex operators carrying zero $Y^{i}$ momentum in the appropriate conformal field theory. In the part of the conformal field theory described by the free scalar fields $Y^{i}$, the correlation functions factorise into the left and the right part, and each part is separately invariant under the rotation group $O(d)$ which acts on these $d$ coordinates. Thus the reduced action has an $O(d) \otimes O(d)$ symmetry, which implies that given a classical solution of the string field theory equations of motion in the subspace carrying zero $Y^{i}$ momemta, we can generate other solutions by acting with this $O(d) \otimes O(d)$ transformation. Of this the diagonal $O(d)$ subgroup simply corresponds to rotating the solution in the $d$ dimensional space, the other generators of $O(d) \otimes O(d)$ acting on the solution produces inequivalent solutions in general, since $O(d) \otimes O(d)$ is not a symmetry of the full action. Although the above argument was given in the context of string field theory, note that the argument is independent of the detailed form of string field theory, and hence the final result is expected to hold for fermionic string theories as well. Note that if one of the coordinates $Y^{m}$ is 
time-like, the $O(d) \otimes O(d)$ transformation gets replaced by $O(d-1,1) \otimes O(d-1,1)$.

The low energy manifestation of this symmetry had been discovered in ref.[3]. We shall briefly reproduce this analysis in a somewhat more general form than the one in which it was discussed in ref.[3]. Let us consider the low energy effective action of string theory in $D$ space-time dimension. This can be obtained either from the study of the $S$-matrix elements in string theory (see ref.[4] and references therein) or from the calculation of the $\beta$-function of the $\sigma$-model [5-8], and is given by,

$$
S=-\int d^{D} x \sqrt{\operatorname{det} G} e^{-\Phi}\left(\Lambda-R^{(D)}(G)+\frac{1}{12} H_{\mu \nu \rho} H^{\mu \nu \rho}-G^{\mu \nu} \partial_{\mu} \Phi \partial_{\nu} \Phi\right)
$$

where $G_{\mu \nu}, B_{\mu \nu}$ and $\Phi$ denote the graviton, the dilaton, and the antisymmetric tensor fields respectively, $H_{\mu \nu \rho}=\partial_{\mu} B_{\nu \rho}+$ cyclic permutations, $R^{(D)}$ denotes the $D$ dimensional Ricci scalar, and $\Lambda$ is the cosmological constant equal to $(D-26) / 3$ for bosonic string and $(D-10) / 2$ for fermionic string. (For simplicity we have set the other background massless fields, which appear in fermionic string theories, to zero.) Let us now split the coordinates $X^{\mu}$ into two sets $\hat{Y}^{m}$ and $\tilde{Y}^{\alpha}(1 \leq m \leq d$, $1 \leq \alpha \leq D-d)$ and consider backgrounds independent of $\hat{Y}^{m}$. Let us further concentrate on backgrounds where $G_{m \alpha}=B_{m \alpha}=0$, i.e. to backgrounds of the form $G=\left(\begin{array}{cc}\hat{G}_{m n} & 0 \\ 0 & \tilde{G}_{\alpha \beta}\end{array}\right), B=\left(\begin{array}{cc}\hat{B}_{m n} & 0 \\ 0 & \tilde{B}_{\alpha \beta}\end{array}\right)$. In this case, after an integration by parts, the action (1) can be shown to take the form:

$$
\begin{gathered}
-\int d^{d} \hat{Y} \int d^{D-d} \tilde{Y} \sqrt{\operatorname{det} \tilde{G}} e^{-\chi}\left[\Lambda-\tilde{G}^{\alpha \beta} \tilde{\partial}_{\alpha} \chi \tilde{\partial}_{\beta} \chi-\frac{1}{8} \tilde{G}^{\alpha \beta} \operatorname{Tr}\left(\tilde{\partial}_{\alpha} M L \tilde{\partial}_{\beta} M L\right)\right. \\
\left.-\tilde{R}^{(D-d)}(\tilde{G})+\frac{1}{12} \tilde{H}_{\alpha \beta \gamma} \tilde{H}^{\alpha \beta \gamma}\right]
\end{gathered}
$$

where,

$$
\begin{gathered}
L=\left(\begin{array}{ll}
0 & 1 \\
1 & 0
\end{array}\right) \\
\chi=\Phi-\ln \sqrt{\operatorname{det} \hat{G}}
\end{gathered}
$$


and,

$$
M=\left(\begin{array}{cc}
\hat{G}^{-1} & -\hat{G}^{-1} \hat{B} \\
\hat{B} \hat{G}^{-1} & \hat{G}-\hat{B} \hat{G}^{-1} \hat{B}
\end{array}\right)
$$

If the coordinates $\hat{Y}^{m}$ are all of Euclidean signature, this action is invariant under an $O(d) \otimes O(d)$ transformation on $\hat{G}, \hat{B}$ and $\Phi$, given by,

$$
\begin{gathered}
M \rightarrow \frac{1}{4}\left(\begin{array}{cc}
S+R & R-S \\
R-S & S+R
\end{array}\right) M\left(\begin{array}{cc}
S^{T}+R^{T} & S^{T}-R^{T} \\
S^{T}-R^{T} & S^{T}+R^{T}
\end{array}\right) \\
\chi \rightarrow \chi, \quad \tilde{G}_{\alpha \beta} \rightarrow \tilde{G}_{\alpha \beta}, \quad \tilde{B}_{\alpha \beta} \rightarrow \tilde{B}_{\alpha \beta}
\end{gathered}
$$

where $S$ and $R$ are $O(d)$ rotation matrices, and $S^{T}, R^{T}$ denote the transpose of the matrices $S, R$. In fact, the action is invariant under a general $O(d, d)$ transformation which leaves the matrix $L$ invariant [3], but the members of the $O(d, d)$ algebra outside the $O(d) \otimes O(d)$ algebra can be shown to generate pure gauge deformations [1] if the coordinates $\hat{Y}^{m}$ are non-compact. The transformations given in eqs.(6), (7) were shown to agree with the $O(d) \otimes O(d)$ transformation on the string fields to linearised order. Corrections to the action given in eq.(1) including higher derivative terms are expected to change the transformation laws given in eqs.(6), (7), but the existence of a modified transformation is guaranteed by the string field theory argument given before.

Since in the above analysis we have explicitly set $G_{m \alpha}$ and $B_{m \alpha}$ to zero, we must make sure that the equations of motion obtained by varying the action with respect to these fields is also $O(d) \otimes O(d)$ invariant. In this case it is easy to see that for the backgrounds considered here these equations of motion are satisfied identically, hence their $O(d) \otimes O(d)$ invariance is obvious.

The above symmetry gets modified to $O(d-1,1) \otimes O(d-1,1)$ when one of the coordinates $\hat{Y}^{m}$ (say $\hat{Y}^{1}$ ) is time-like. In this case the transformation laws (5) 
get modified to,

$$
M \rightarrow \frac{1}{4}\left(\begin{array}{cc}
\eta(S+R) \eta & \eta(R-S) \\
(R-S) \eta & S+R
\end{array}\right) M\left(\begin{array}{cc}
\eta\left(S^{T}+R^{T}\right) \eta & \eta\left(R^{T}-S^{T}\right) \\
\left(R^{T}-S^{T}\right) \eta & \left(S^{T}+R^{T}\right)
\end{array}\right)
$$

where $\eta=\operatorname{diag}(-1,1, \ldots 1)$, and $S$ and $R$ are $O(d-1,1)$ matrices satisfying,

$$
S \eta S^{T}=\eta, \quad R \eta R^{T}=\eta
$$

In all the examples we shall consider, one of the coordinates $\hat{Y}^{m}$ will be timelike, and hence the relevant group will be $O(d-1,1) \otimes O(d-1,1)$. We shall now examine the transformation laws of various fields under the $O(d-1,1) \otimes O(d-1,1)$ group in some detail. In component form the transformed fields $\hat{G}_{i j}^{\prime}, \hat{B}_{i j}^{\prime}$ and $\Phi^{\prime}$ are given by,

$$
\begin{aligned}
\left(\hat{G}^{\prime-1}\right)_{i j}= & \frac{1}{4}\left[\eta(S+R) \eta \hat{G}^{-1} \eta\left(S^{T}+R^{T}\right) \eta+\eta(R-S)\left(\hat{G}-\hat{B} \hat{G}^{-1} \hat{B}\right)\left(R^{T}-S^{T}\right) \eta\right. \\
& \left.-\eta(S+R) \eta \hat{G}^{-1} \hat{B}\left(R^{T}-S^{T}\right) \eta+\eta(R-S) \hat{B} \hat{G}^{-1} \eta\left(S^{T}+R^{T}\right) \eta\right]_{i j} \\
\hat{B}_{i j}^{\prime}= & \frac{1}{4}\left(\left[(R-S) \eta \hat{G}^{-1} \eta\left(S^{T}+R^{T}\right) \eta+(S+R)\left(\hat{G}-\hat{B} \hat{G}^{-1} \hat{B}\right)\left(R^{T}-S^{T}\right) \eta\right.\right. \\
& \left.\left.+(S+R) \hat{B} \hat{G}^{-1} \eta\left(S^{T}+R^{T}\right) \eta-(R-S) \eta \hat{G}^{-1} \hat{B}\left(R^{T}-S^{T}\right) \eta\right] \hat{G}^{\prime}\right)_{i j} \\
\Phi^{\prime}= & \Phi-\frac{1}{2} \ln \operatorname{det} \hat{G}+\frac{1}{2} \ln \operatorname{det} \hat{G}^{\prime}
\end{aligned}
$$

The transformation laws of $\hat{G}$ and $\hat{B}$ may be expressed in a compact form by defining the matrix:

$$
C \equiv \hat{G}-\eta \hat{G}^{-1} \eta-\hat{B} \hat{G}^{-1} \hat{B}+\hat{B} \hat{G}^{-1} \eta+\eta \hat{G}^{-1} \hat{B}
$$

The transformation law of $C$ then takes a simple form:

$$
C^{\prime}=S C R^{T}
$$

Let us now turn to specific examples. We start from a simple solution of the 
equation of motion (1) of the form [9] [10]:

$$
\begin{aligned}
d s^{2} & =-\tanh ^{2} \frac{Q r}{2} d t^{2}+d r^{2}+\sum_{i=1}^{d-1} d X^{i} d X^{i} \\
B_{\mu \nu} & =0 \\
\Phi & =-\ln \cosh ^{2} \frac{Q r}{2}+\Phi_{0}
\end{aligned}
$$

where $\Phi_{0}$ is an arbitrary parameter, and,

$$
\begin{aligned}
Q=\sqrt{-\Lambda} & =\sqrt{\frac{25-d}{3}} \text { for bosonic string } \\
& =\sqrt{\frac{9-d}{2}} \text { for fermionic string }
\end{aligned}
$$

Since the solution is independent of $d-1$ space-like coordinates, it can be called a $d-1$ brane solution in the language of ref.[12]. Note that a priori we cannot ignore the contribution from the higher derivative terms in the action since the scale of spatial variation of the solution is set by $Q$ which is of order 1 . This problem may be avoided if instead of just taking $d-1$ scalar (super)-fields $X^{i}$, we take $d-1$ scalar (super)-fields together with a (super)-conformal field theory of central charge $c\left(\frac{3}{2} c\right)$. In this case $Q$ will be given by,

$$
\begin{aligned}
Q & =\sqrt{\frac{25-d-c}{3}} \text { for bosonic string } \\
& =\sqrt{\frac{9-d-c}{2}} \text { for fermionic string }
\end{aligned}
$$

and we may consider a situation where $d+c \simeq 25(d+c \simeq 9)$ so that $Q$ is small. In this case the effect of higher derivative terms in the effective action will be small, at least away from any singularity.

\footnotetext{
$\star$ The $O(d-1,1) \otimes O(d-1,1)$ transformation may also be applied to the more general class of solutions discussed in ref.[11], but we shall not discuss it here.
} 
The solution given in eq.(13) has the property that it is independent of the coordinates $t$ and $X^{i}$. Thus we can make an $O(d-1,1) \otimes O(d-1,1)$ transformation on the fields so that the new configuration will also be a solution of the equations of motion. For the background defined in eq.(13) the tensor $C_{i j}$ defined in eq.(11) takes the form:

$$
C_{i j}=-\delta_{i 1} \delta_{j 1}\left(\tanh ^{2} \frac{Q r}{2}-\operatorname{coth}^{2} \frac{Q r}{2}\right)
$$

Thus from eq.(12) we get,

$$
C_{i j}^{\prime}=-S_{i 1} R_{j 1}\left(\tanh ^{2} \frac{Q r}{2}-\operatorname{coth}^{2} \frac{Q r}{2}\right)
$$

In order to study the set of inequivalent classical solutions, we note that under a Lorentz transformation, $C^{\prime} \rightarrow M C^{\prime} M^{T}$ where $M$ is an $O(d-1,1)$ matrix. This corresponds to changing $S$ to $M S$ and $R$ to $M R$. Thus,

$$
S_{i 1} \rightarrow M_{i k} S_{k 1}, \quad R_{i 1} \rightarrow M_{i k} R_{k 1}
$$

Both the vectors $S_{i 1}$ and $R_{i 1}$ are normalised to -1 with respect to the metric $\eta$ since they form the first columns of $O(d-1,1)$ matrices. Thus by choosing an appropriate $M$ we may bring the vectors $S_{i 1}$ and $R_{j 1}$ into the form:

$$
\begin{gathered}
S_{11}=\cosh \theta, \quad S_{21}=-\sinh \theta, \quad S_{i 1}=0 \text { for } i \geq 3 \\
R_{11}=\cosh \theta, \quad R_{21}=\sinh \theta, \quad R_{i 1}=0 \quad \text { for } i \geq 3
\end{gathered}
$$

For this choice of $S$ and $R$ eq.(10) gives the following form of the transformed fields 
$\hat{G}^{\prime}, \hat{B}^{\prime}$ and $\Phi^{\prime}$ :

$$
\begin{aligned}
& \hat{G}^{\prime}=\left(\begin{array}{ccccc}
-\frac{\sinh ^{2}(Q r / 2)}{\cosh ^{2}(Q r / 2)+\sinh ^{2} \theta} & 0 & 0 & \cdots & 0 \\
0 & \frac{\cosh ^{2}(Q r / 2)}{\cosh ^{2}(Q r / 2)+\sinh ^{2} \theta} & 0 & \cdots & 0 \\
0 & 0 & 1 & \cdots & 0 \\
\cdot & \cdot & . & \cdots & . \\
\cdot & \cdot & . & \cdots & . \\
0 & 0 & 0 & \cdots & 1
\end{array}\right) \\
& \hat{B}^{\prime}=\frac{\cosh \theta \sinh \theta}{\cosh ^{2}(Q r / 2)+\sinh ^{2} \theta}\left(\begin{array}{ccccc}
0 & 1 & 0 & \cdots & 0 \\
-1 & 0 & 0 & \cdots & 0 \\
0 & 0 & 0 & \cdots & 0 \\
\cdot & . & . & \cdots & . \\
\cdot & . & . & \cdots & \cdot \\
0 & 0 & 0 & \cdots & 0
\end{array}\right) \\
& \Phi^{\prime}=-\ln \left(\cosh ^{2}(Q r / 2)+\sinh ^{2} \theta\right)+\Phi_{0}
\end{aligned}
$$

Thus the full metric now takes the form:

$$
\begin{aligned}
d s^{2}= & -\frac{\sinh ^{2}(Q r / 2)}{\cosh ^{2}(Q r / 2)+\sinh ^{2} \theta} d t^{2}+d r^{2}+\frac{\cosh ^{2}(Q r / 2)}{\cosh ^{2}(Q r / 2)+\sinh ^{2} \theta}\left(d X^{1}\right)^{2} \\
& +\sum_{i=2}^{d-1} d X^{i} d X^{i}
\end{aligned}
$$

and has a coordinate singularity at $r=0$. This singularity may be removed by choosing a new coordinate system:

$$
u=\sinh (Q r / 2) e^{Q t /(2 \cosh \theta)}, \quad v=\sinh (Q r / 2) e^{-Q t /(2 \cosh \theta)}
$$


In this coordinate system the metric takes the form:

$$
\begin{aligned}
d s^{2}= & \frac{2}{Q^{2}} d u d v\left(\frac{\cosh ^{2} \theta}{u v+\cosh ^{2} \theta}+\frac{1}{u v+1}\right) \\
& -\frac{1}{Q^{2}} \frac{\sinh ^{2} \theta}{(u v+1)\left(u v+\cosh ^{2} \theta\right)}\left(v^{2} d u^{2}+u^{2} d v^{2}\right) \\
& +\frac{u v+1}{u v+\cosh ^{2} \theta}\left(d X^{1}\right)^{2}+\sum_{i=2}^{d-1} d X^{i} d X^{i}
\end{aligned}
$$

Thus we see that at $r=0$, i.e. at $u=0$ or $v=0$, the metric does not have any singularity in this new coordinate system. In fact, in this coordinate system the metric has finite non-zero eigenvalues in the region $u v>-1, u, v$ finite. In order to make sure that the solution is non-singular at $r=0$, we must also check that the dilaton, as well as the field strength associated with the anti-symmetric tensor field are non-singular at $r=0$. The dilaton given in eq.(20) is clearly nonsingular at $r=0$. The antisymmetric tensor field strength $H_{\mu \nu \rho}=\left(\partial_{\mu} B_{\nu \rho}+\right.$ cyclic permutations) takes the form:

$$
H_{r t 1}=\partial_{r} B_{t 1}=\frac{-Q \cosh \theta \sinh \theta \cosh (Q r / 2) \sinh (Q r / 2)}{\left(\cosh ^{2}(Q r / 2)+\sinh ^{2} \theta\right)^{2}}
$$

When transformed to the $u-v$ coordinate system, this becomes,

$$
H_{u v 1}=\frac{2}{Q} \frac{\cosh ^{2} \theta \sinh \theta}{\left(u v+\cosh ^{2} \theta\right)^{2}}
$$

which is clearly non-singular at $u=0$ or $v=0$.

So far we have discussed the part of $O(d-1,1) \otimes O(d-1,1)$ transformation that is connected to identity. As was discussed in ref.[1], the effect of the disconnected part of the $O(d) \otimes O(d)$ transformation on the solution is to expand the solution in powers of $e^{Q r}$ and reverse the sign of all the odd powers of $e^{Q r}$. This transforms 
the solution given in eq.(20) to,

$$
\begin{aligned}
& \hat{G}^{\prime}=\left(\begin{array}{ccccc}
-\frac{\cosh ^{2}(Q r / 2)}{\sinh ^{2}(Q r / 2)-\sinh ^{2} \theta} & 0 & 0 & \cdots & 0 \\
0 & \frac{\sinh ^{2}(Q r / 2)}{\sinh ^{2}(Q r / 2)-\sinh ^{2} \theta} & 0 & \cdots & 0 \\
0 & 0 & 1 & \cdots & 0 \\
\cdot & \cdot & \cdot & \cdots & \cdot \\
\cdot & \cdot & \cdot & \ldots & \cdot \\
0 & 0 & 0 & \cdots & 1
\end{array}\right) \\
& \hat{B}^{\prime}=-\frac{\cosh \theta \sinh \theta}{\sinh ^{2}(Q r / 2)-\sinh ^{2} \theta}\left(\begin{array}{ccccc}
0 & 1 & 0 & \cdots & 0 \\
-1 & 0 & 0 & \cdots & 0 \\
0 & 0 & 0 & \cdots & 0 \\
\cdot & . & . & \cdots & \cdot \\
\cdot & . & . & \cdots & . \\
0 & 0 & 0 & \cdots & 0
\end{array}\right) \\
& \Phi^{\prime}=-\ln \left(\sinh ^{2}(Q r / 2)-\sinh ^{2} \theta\right)+\Phi_{0}
\end{aligned}
$$

Thus in this case the solution is singular at $r=2 \theta / Q$.

As was shown by Witten [10] (see also refs.[13 - 17] ), for the solution given in eq.(13), after modification due to higher order terms have been taken into account, the coordinates $r$ and $t$ together describe a solvable conformal field theory based on the gauged SL(2,R) WZW theory, where a non-compact subgroup of $\operatorname{SL}(2, \mathrm{R})$ is gauged. The level $k$ of the WZW theory is related to $Q$ by the relation:

$$
2+3 Q^{2}=\frac{3 k}{k-2}-1
$$

so that the $Q \rightarrow 0$ limit corresponds to $k \rightarrow \infty$. It is thus natural to ask whether there are solvable conformal field theories which correspond to the solution given in eq.(20). In fact, conformal field theories associated with Euclidean continuation of these solutions have been found in ref.[18] by gauging a linear combination of a compact $\mathrm{U}(1)$ generator of $\mathrm{SL}(2, \mathrm{R})$ and the $\mathrm{U}(1)$ currents generated by $\partial X^{i}$, $\bar{\partial} X^{i}$. By taking independent linear combinations of the $\mathrm{U}(1)$ currents in the left 
and the right sector one can get the Euclidean continuation of the background given in eq.(20) (with $\theta$ replaced by $i \theta$ ). This is not surprising, since the original $O(d) \otimes O(d)$ symmetry was due to the freedom of independent rotations in the left and the right sector.

Note that if we take some of the directions $X^{i}$ to be compact, then even the diagonal $O(d-1,1)$ subgroup, acting on the solution, generates new solutions, since this is no longer a symmetry of the full theory. This has been exploited in ref.[18] to get new exact solutions of string theory. In the analysis of this paper we shall restrict ourselves to the non-compact case.

We shall now consider a second example, where the solution, in general, does not correspond to a solvable conformal field theory. This is the five-brane solution of the low energy effective field theory in ten dimensional superstring or heterotic string theory (or, equivalently, the 21-brane solution in 26 dimensional bosonic string theory) described in ref.[12]. (See also refs.[19 - 29] for related work.) In a particular coordinate system the solution takes the form [20]:

$$
\begin{aligned}
d s^{2} & =-\tanh ^{2} r d t^{2}+\left[M+\delta^{2}\left(\cosh ^{2} r-\frac{1}{2}\right)\right]\left(d r^{2}+d \Omega_{3}^{2}\right)+\sum_{i=1}^{5} d X^{i} d X^{i} \\
\Phi & =\ln \left(\frac{M+\delta^{2}\left(\cosh ^{2} r-\frac{1}{2}\right)}{\delta^{2} \cosh ^{2} r}\right)+\Phi_{0} \\
H & =2 Q_{0} \epsilon_{3}
\end{aligned}
$$

where $M$ and $\Phi_{0}$ are independent continuous parameters,

$$
Q_{0} \equiv \sqrt{M^{2}-\frac{1}{4} \delta^{4}}
$$

is quantised, $d \Omega_{3}$ is the line element on the 3-sphere, and $\epsilon_{3}$ is the volume form on the same 3-sphere. (For bosonic string theory the sum over $i$ runs from 1 to 21

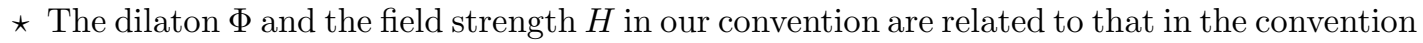
of ref.[12] by a factor of 2 .
} 
instead of from 1 to 5.) Although the solution does not appear to be flat in the asymptotic region $r \rightarrow \infty$, after a coordinate transformation:

$$
y=\delta \cosh r
$$

one gets a metric which reduces to 10 dimensional Minkowski metric in the region $r \rightarrow \infty$.

Let us note that the solution is independent of the coordinates $t$ and $X^{i}$. Thus, as before, we can construct new solutions through $O(d-1,1) \otimes O(d-1,1)$ transformation (here $d=6$ ). Counting of independent parameters labelling the transformed solution proceeds exactly in the same way as before, and we are left with one independent parameter $\theta$. The new solution obtained in this way is given by:

$$
\begin{aligned}
d s^{2}= & -\frac{\sinh ^{2} r}{\cosh ^{2} r+\sinh ^{2} \theta} d t^{2}+\frac{\cosh ^{2} r}{\cosh ^{2} r+\sinh ^{2} \theta}\left(d X^{1}\right)^{2} \\
& +\left(M+\delta^{2}\left(\cosh ^{2} r-\frac{1}{2}\right)\right)\left(d r^{2}+d \Omega_{3}^{2}\right)+\sum_{i=2}^{5} d X^{i} d X^{i} \\
\Phi= & \ln \frac{M+\delta^{2}\left(\cosh ^{2} r-\frac{1}{2}\right)}{\delta^{2}\left(\cosh ^{2} r+\sinh ^{2} \theta\right)}+\Phi_{0} \\
H^{(i n t)}= & 2 Q_{0} \epsilon_{3} \\
\hat{B}= & \frac{\cosh ^{2} \sinh \theta}{\cosh ^{2} r+\sinh ^{2} \theta}\left(\begin{array}{ccccc}
0 & 1 & 0 & \cdots & 0 \\
-1 & 0 & 0 & \cdots & 0 \\
0 & 0 & 0 & \cdots & 0 \\
\cdot & . & . & \cdots & . \\
. & . & . & \cdots & . \\
0 & 0 & 0 & \cdots & 0
\end{array}\right)
\end{aligned}
$$

where $H^{(i n t)}$ denotes the field strength associated with the antisymmetric tensor field in the internal 3-sphere, and $\hat{B}$ denotes the components of the antisymmetric tensor field in the six dimensional space spanned by the coordinates $t$ and $X^{i}$. As before, it is easy to see that the point $r=0$ represents a coordinate singularity, and 
the field strength associated with the antisymmetric tensor field is regular at $r=0$. Thus we see that using the $O(d-1,1) \otimes O(d-1,1)$ transformation we can generate a solution of the low energy effective field theory equations of motion characterised by three continuous parameters $\Phi_{0}, M$ and $\theta$, and one discrete parameter $Q_{0}$.

In conclusion, we have demonstrated in this paper that the $O(d) \otimes O(d)(O(d-$ $1,1) \otimes O(d-1,1))$ symmetry may be used effectively to generate new classical solutions in string theory from the known ones. Although we have considered only a few examples, it is clear that this transformation can be applied on other known classical solutions as well. It will be interesting to study the the physical consequences of continuous parameter family of solutions in string theory implied by the $O(d) \otimes O(d)$ symmetry, particularly on the cosmology of the early universe in string theory [30].

Finally, note that for suitable backgrounds, the $O(d-1,1) \otimes O(d-1,1)$ symmetry of the reduced action may be extended to $O(d-1,1) \otimes O(d+15,1)$ symmetry in the case of heterotic string theory, due to the possibility of including the 16 internal coordinates in the rotation. This rotation, in general, will produce a charged black hole [21] from an uncharged one. Thus besides producing new solutions, our method also opens up the possibility of relating different known solutions in string theory by the twisting procedure. 


\section{REFERENCES}

N1 A.Sen preprint IC/91/195 (TIFR-TH-91-35). N2 G. Veneziano preprint CERN-TH-6077/91. N3 K. Meissner and G. Veneziano, preprint CERN-TH6138/91. N4 J. Schwarz, Phys. Rep. 89 (1982) 223. N5 C. Lovelace, Phys. Lett. 135B (1984) 75. N6 E. Fradkin and A. Tseytlin, Phys. Lett. 158B (1985) 316. N7 A. Sen, Phys. Rev. D32 (1985) 2102; Phys. Rev. Lett. 55 (1985) 1846. N8

C.G. Callan, D. Friedan, E. Martinec and M. Perry, Nucl. Phys. B262 (1985) 593. N9 G. Mandal, A.M. Sengupta and S.R. Wadia, preprint IASSNS-HEP-91/10. N10 E. Witten, preprint IASSNS-HEP-91/12. N11 S. Mukherji, S. Mukhi and A. Sen, preprint TIFR/TH/91-28. N12 G. Horowitz and A. Strominger, Nucl. Phys. B360 (1991) 197. N13 S. Elitzur, A. Forge and E. Rabinovici, Preprint RI-143-90. N14 K. Bardacki, M. Crescimannu, and E. Rabinovici, Nucl. Phys. B344 (1990) 344. N15 M. Rocek, K. Schoutens and A. Sevrin, preprint IASSNS-HEP-91/14. N16 R. Dijkgraaf, E. Verlinde and H. Verlinde, preprint PUPT-1252, IASSNSHEP-91/22. N17 I. Bars and D. Nemeschansky, Nucl. Phys. B348 (1991) 89. N18 N. Ishibashi, M. Li and A.R. Steif, preprint UCSB-91-28. N19 C. G. Callan, J. Harvey and A. Strominger, Nucl. Phys. B359 (1991) 611. N20 S. Giddings and A. Strominger, preprint UCSBTH-91-35. N21 D. Garfinkle, G. Horowitz and A. Strominger, preprint UCSB-TH-90-66. N22 A. Dabholkar, G. Gibbons, J. Harvey and F. Ruiz, Nucl. Phys. B340 (1990) 33. N23 M.J. Duff and J. Lu, preprint CTPTAMU-81/90. N24 C. G. Callan, R. C. Myers and M. Perry, Nucl. Phys. B311 (1988) 673. N25 R.C. Myers, Nucl. Phys. B289 (1987) 701. N26 G. Gibbons, Nucl. Phys. B207 (1982) 337; G. Gibbons and K. Maeda, Nucl. Phys. B298 (1988) 741. N27 H.J. de Vega and N. Sanchez, Nucl. Phys. B309 (1988) 552. N28 P. Mazur, Gen. Rel. and Grav. 19 (1987) 1173. N29 R. C. Myers and M. Perry, Ann. Phys. 172 (1986) 304. N30 R. Brandenberger and C. Vafa, Nucl. Phys. B316 (1989) 391. 